\title{
MOOCs in der Wirtschaftsinformatik
}

\section{Oliver Bendel}

Eingegangen: 30. Oktober 2014 / Angenommen: 13. Dezember 2014 / Online publiziert: 21. Dezember 2014 (C) Springer Fachmedien Wiesbaden 2014

Zusammenfassung Der vorliegende Beitrag erklärt den Begriff der MOOCs, arbeitet deren Merkmale heraus und liefert einen Überblick über Angebote, Geschäftsmodelle und Leistungsnachweise. Untersucht wird, inwieweit die Wirtschaftsinformatik von der Bewegung betroffen ist und ob Interessierte und Studierende sich auf diese Weise auf Hochschulniveau aus- und weiterbilden können. Es besteht, wie sich herauskristallisiert, ein schmales, aber nützliches Angebot, als mögliche Ergänzung zum bisherigen Unterricht.

Schlüsselwörter E-Learning $\cdot$ MOOC $\cdot$ Ausbildung $\cdot$ Weiterbildung · Wirtschaftsinformatik · Informatik

\section{Ein vieldiskutiertes Phänomen}

MOOCs sind leicht zugängliche und doch anspruchsvolle Lernumgebungen. In den 2010er-Jahren wurden sie zum vieldiskutierten Phänomen (vgl. Schulmeister 2013, 17 ff.). Der vorliegende Beitrag erklärt den Begriff der MOOCs, arbeitet deren Ziele und Merkmale heraus und gibt einen Überblick über aktuelle Angebote und künftige Geschäftsmodelle sowie mögliche Leistungsnachweise. Insbesondere wird untersucht, inwieweit die Wirtschaftsinformatik (WI) von der Bewegung betroffen ist, genauer ob Interessierte und Studierende sich auf diese Weise auf Hochschulniveau aus- und weiterbilden können, ob die Kurse im Studium angerechnet werden oder ob sie einen anderweitigen Nutzen haben und ob WI-Ausbildung und Hochschullehre

O. Bendel $(\square)$

Hochschule für Wirtschaft, Institut für Wirtschaftsinformatik,

Fachhochschule Nordwestschweiz, Bahnhofstrasse 6,

5210 Windisch, Schweiz

E-Mail: oliver.bendel@fhnw.ch 
dadurch profitieren können oder ob sie gefährdet sind. Der Verfasser stützt sich auf Literaturanalyse, die kritische Auswertung von Websites sowie eigene Beobachtungen und Erfahrungen.

\section{Grundlagen von MOOCs}

Fokussiert wird auf den Begriff, den Hintergrund und die Entwicklung sowie die Merkmale und Funktionen von MOOCs. Ziele sind eine grobe Systematisierung und eine klare Vorstellung von den Möglichkeiten und Grenzen.

\subsection{Begriff, „MOOC“}

Ein MOOC, ein Massive Open Online Course, ist ein internetbasierter Kurs, der sich an eine große Menge von Teilnehmenden richtet (engl. „massive“: „riesig, enorm“), offen für alle Interessierten (engl. „open“) und in der Durchführung meist kostenlos ist (vgl. Bendel 2014). Man unterscheidet zwischen xMOOCs und cMOOCs. ${ }^{1}$ Ein Äquivalent in der deutschen Sprache hat sich nicht etabliert. Eine besondere Form oder Gegenform - sind die Small Private Online Courses (SPOCs), eher kleine und geschlossene Kurse, die allerdings auf der MOOC-Technologie basieren. Corporate MOOCs richten sich an Mitarbeitende von Unternehmen. Damit sind sie eigentlich nicht „offen“; tendenziell werden sie aber in Großkonzernen mit zahlreichen Partizipierenden eingesetzt.

MOOCs gehören zum E-Learning. Hierbei handelt es sich um ein Lernen, das mit Informations- und Kommunikationstechnologien (Basis- und Lerntechnologien) und darauf aufbauenden Lernsystemen unterstützt wird (vgl. Bendel und Stoller-Schai 2001). Auch die Realisierung als Mobile Learning ist möglich (vgl. Bendel 2011). MOOCs eignen sich in der Regel für Tablets und Notebooks. Ob Aufgaben und Feedbacks über Smartphones effizient und effektiv abgewickelt werden können, sei dahingestellt. Blended Learning als Kombination von konventionellem und virtuellem Lernen ist innerhalb der MOOCs kaum anzutreffen. Natürlich kann man diese in die Präsenzlehre einbetten. Der Anbieter Coursera wirbt auf seiner Website: „Many of our partner institutions are using our online platform to provide their on-campus students with an improved learning experience. This blended model of learning has shown in studies to increase student engagement, attendance and performance." (Website Coursera) Es wird allerdings nicht einfach sein, MOOCs mit dem bestehenden konventionellen und virtuellen Betrieb zu vereinen, da sie mehr oder weniger abgeschlossen und eigenständig sind.

\subsection{Hintergrund und Entwicklung der MOOCs}

Vorläufer von MOOCs traten bereits um die Jahrtausendwende auf. Im deutschsprachigen Raum experimentierten Hochschulen mit Formen, die Videos und Folien

\footnotetext{
${ }^{1}$ „,X“ steht für „,extension“ (die Harvard University machte mit diesem Buchstaben in ihren Verzeichnissen auf virtuelle Kurse aufmerksam), „c“ für „connectivism“.
} 
integrieren und den heutigen xMOOCs ähneln. Ein Beispiel sind die Kurse der Virtuellen Hochschule Oberrhein (VIROR), u. a. zu Informatik, Psychologie, Medizin und Ingenieurwesen. Ab Mitte 1998 wurden diese von badischen Universitäten aufgebaut (vgl. Kandzia 1999). Weltweit offerieren Hochschulen, Bildungseinrichtungen und Mittler immer mehr MOOCs und erreichen immer mehr Studierende. So gab es beispielsweise an der ETH Lausanne (EPFL) 2012 über 50.000 Anmeldungen für deren ersten MOOC, den Informatikkurs „Scala“ (vgl. Güntert 2013), und Coursera führte 2013 den Kurs „Gamification“, wie man diesem selbst entnehmen durfte, mit ca. 70.000 Teilnehmerinnen und Teilnehmern durch. Mit den akademischen Erstellern und aktuellen Konzepten hängt zusammen, dass wissenschaftliche Inhalte dominieren.

\subsection{Merkmale und Funktionen von MOOCs}

Massive Open Online Courses erreichen potenziell Menschen mit unterschiedlichem Bildungshintergrund und Wissensstand. Tatsächlich dominieren jedoch bereits gut Ausgebildete (vgl. Güntert 2013). MOOCs werden vielfach als Möglichkeit zur Weiterbildung auf hohem Niveau wahrgenommen. Dabei greift vor allem die interessierte Einzelperson zu, ob aus persönlichem oder beruflichem Interesse. Dem entgegensteuern könnte eine stärkere Anbindung an den klassischen Hochschulbetrieb unter Berücksichtigung des Bologna-Prozesses, wie es z. B. iversity mit seinen ECTS-Kursen versucht: Damit würden die MOOCs im Rahmen der akademischen Aus- und Weiterbildung gestärkt und die „normalen“ Studierenden motiviert, daran teilzunehmen.

Nicht alle Lernenden halten bis zum Schluss durch. Nur ca. $20 \%$ absolvierten im Kurs „Scala“ die Abschlussprüfung (vgl. Güntert 2013). Der Vergleich mit Studienund Kursabbrüchen hinkt, da die Teilnehmenden in der Regel bereits eine Ausbildung und die MOOCs einen wenig verpflichtenden Charakter haben. Die Gründe für das vorzeitige Beenden mögen vielfältig sein. Es können didaktische Schwächen bei den Kursen vorhanden sein, schlecht funktionierende Kommunikationswerkzeuge, unklare Beschreibungen und ständige Überforderungen (vgl. Colman 2013). Bei den Lernenden sind mangelnde Erfahrungen, ungenügende Selbstdisziplin und nichterfüllte Erwartungen möglich (vgl. Bendel 2014). Sie können das Gefühl haben, zu viel Zeit investieren zu müssen und ungenügendes Wissen zu haben (vgl. Colman 2013).

Bei den großen Anbietern überwiegen xMOOCs. Diese sind, wie eine kursorische Sichtung zeigt, eine Kombination aus Syllabus (Verzeichnis), Lehrvideos, Dokumenten, Folien, Assessments (z. B. zur Selbsteinschätzung) und Assignments (Aufgaben), standardisierten Beurteilungsbogen sowie Kommunikationswerkzeugen und sozialen Medien. Am Ende der Durchführung, die Wochen oder Monate dauert, findet auf Wunsch eine Zertifizierung statt. Im Verzeichnis wird der Kurs meist wochenweise strukturiert. Die Lehrvideos entstehen aus der Aufzeichnung von konventionellem Unterricht oder einer speziellen Aufnahme unter Studiobedingungen. Die Folien werden bevorzugt aus Präsentationsanwendungen generiert und in die Lernumgebung eingebunden (z. B. werden Dozent und Folien abwechselnd gezeigt) oder als Downloads bereitgestellt. Die Selbsttests konstituieren sich mehrheitlich aus Single- und Multiple-Choice-Fragen und Lückentexten, sodass sie automatisiert 
ausgewertet werden können. Bei den Tests finden sich auch offene Fragen. Als Kommunikationskanäle sind integrierte Diskussionsforen (etwa zu jeder Lerneinheit) und Chats verbreitet.

Wegen der hohen Teilnehmerzahlen muss eine ausreichende, stabile und sichere technische Infrastruktur aufgebaut werden. Zudem sind spezielle Bewertungs- und Feedbackmechanismen notwendig. Ein xMOOC, der eher lehrerzentriert und formell ist, stößt schnell an Grenzen, es sei denn, man gestaltet ihn mit Peer-Review-Prozessen, wobei die Peers die anderen Teilnehmenden sind. Ein direktes, persönliches Feedback durch die Lehrenden ist nur ausnahmsweise möglich. Einige Dozierende und Anbieter beschäftigen Tutoren, die eine gewisse Menge an Anfragen und Leistungen bewältigen können. Ein cMOOC, der eher lernerzentriert, informell und von partizipativen Medien bestimmt ist, kennt prinzipiell keine Überlastung, stellt aber wegen der fehlenden zentralen Organisation und der fehlenden verbindlichen Vorgaben hohe Anforderungen an den Lernenden, der sich selbst anleiten, zielorientiert vorgehen und sich permanent mit anderen verständigen muss. SPOCs sind restriktiver organisiert und verlangen nach Berechtigungen und Zulassungen. Man kann sie gut im Hochschul- und Unternehmensbereich und überhaupt in modularen Systemen verwenden.

\section{Anbieter und Plattformen für MOOCs}

Aufgeführt werden wichtige US-amerikanische, deutsche und schweizerische Anbieter von MOOCs, unter vereinzelter Nennung von Nutzerzahlen. Andere Länder können aus Platzgründen nicht berücksichtigt werden. Bei den USA wird kurz auf die Inhalte eingegangen, beim deutschsprachigen Raum darauf verzichtet, da sonst Redundanzen zum nächsten Kapitel vorhanden wären.

\subsection{Anbieter und Plattformen in den USA}

In den USA gibt es eine beachtliche Zahl von Kooperationen und Plattformen. Harvard University und Massachusetts Institute of Technology (MIT) haben das Joint Venture edX (www.edx.org) ins Leben gerufen. Die 1,3 Millionen Benutzer (vgl. Becker und Rojas 2014) finden fast alles, von Sprachkursen über Geschichtsvorlesungen bis hin zu Technik- und Informatikunterricht. Coursera (www.coursera.org) ist ein Mittler für englisch-, aber auch deutschsprachige Inhalte. Das Angebot ist stark ausdifferenziert und wird von ca. 5 Millionen genutzt (vgl. Becker und Rojas 2014). Udacity (www.udacity.com) ist spezialisierter und bietet $u$. a. Informatikkurse (Data Science, Web Development, Software Engineering etc.) an, bei 750.000 Lernenden bis 2012 (vgl. Becker und Rojas 2014). Die Khan Academy von Salman Khan, die in diesem Zusammenhang oft genannt wird, arbeitet hauptsächlich mit Video Tutorials, ist also kaum zu den MOOC-Vertretern zu zählen.

\subsection{Anbieter und Plattformen im deutschsprachigen Raum}

Im deutschsprachigen Raum offerieren u. a. LMU München, TU München, ETH Lausanne und Universität Zürich (UZH) wissenschaftliche MOOCs, z. B. via Coursera. 
Dadurch wird die virtuelle Lehre in Deutschland und in der Schweiz erneut gestärkt. Sie hatte in den 00er-Jahren finanzielle Förderungen erfahren, durch Europäische Union, Bundesministerium für Bildung und Forschung (BMBF) und Schweizer Nationalfonds (SNF, mit seinem Swiss Virtual Campus, 2000 bis 2007/08). iversity (www.iversity.org) aus Berlin ist ein kommerzieller Mittler mit einer breiten Palette an Kursen und 330.000 eingeschriebenen Nutzern mit Stand Ende Januar 2014 (vgl. Becker und Rojas 2014). Die Plattform OpenCourseWorld (www.opencourseworld. de) der IMC AG, eines LMS-Unternehmens, hat ein schmales Angebot. openHPI (open.hpi.de) wird vom Hasso-Plattner-Institut (HPI) für Softwaresystemtechnik betrieben, mit einem engen Fokus. Dabei erreichte man bis 201453.000 Teilnehmer aus 114 Ländern (vgl. Becker und Rojas 2014). openSAP (open.sap.com), genutzt von ca. 150.000 Lernenden, ist von SAP, mit SAP-Produkten als Schwerpunkt und in Kooperation mit dem HPI (Website openSAP). ${ }^{2}$

\subsection{Geschäftsmodelle und Leistungsnachweise}

Die Anmeldung zum MOOC ist meist gratis, die Durchführung ebenfalls, die Zertifizierung kostenpflichtig, wenn sie personalisiert ist. Udacity nimmt „,bis zu 89 Dollar für ein Zertifikat“ (Schmidt 2013), Coursera „zwischen 30 und 100 Dollar“ (Becker und Rojas 2014). Die Kurse werden wie klassische Produkte und Dienstleistungen verkauft und vertrieben (Information, Werbung, Anreize für das Werben von Contentanbietern und -nachfragern, Rabatte für die Zertifizierung, Bereitstellung des Zugangs), und die Plattformen registrieren und verwerten die persönlichen Daten und die Eingaben der Studierenden. Für eine Hochschule können MOOCs betriebswirtschaftlich sinnvolle Gefäße und Maßnahmen sein. Sie mögen insbesondere dort, wo Hunderte Studierende in einer Vorlesung sitzen, zur Entlastung der Hörsäle und zu finanziellen Einsparungen führen. Es sind allerdings gewisse Investitionen erforderlich. Dabei sind die direkten Kosten ebenso wie organisationale und persönliche Doppelbelastungen zu berücksichtigen.

Die IMC-Plattform wendet sich einerseits an Privatpersonen, die für das Zertifikat bezahlen, andererseits an Hochschulen, Forschungseinrichtungen, Coaches und Unternehmen, die selbst kostenfrei Kurse einbringen können (vgl. Website OpenCourseWorld). openHPI ist für ein breites und ein Fachpublikum gleichermaßen gedacht. Die Teilnahme ist kostenlos und an keine Voraussetzungen gebunden (vgl. Website openHPI). iversity arbeitet nach eigenen Angaben eng mit Dozierenden, Hochschulen und Bildungseinrichtungen zusammen, um Kurse zu produzieren, die interaktiv sind und Spaß machen. Man sei ,,auf der Suche nach Lösungen, die für Bildungseinrichtungen, Studierende und unsere Gesellschaft gleichermaßen gut funktionieren und für alle einen Mehrwert schaffen“ (Website iversity). Studierende werden dazu animiert, geeignete Lehrende zu melden und für iversity zu gewinnen.

Coursera kooperiert, so die Selbstauskunft, mit führenden Universitäten und Organisationen auf der ganzen Welt, um Onlinekurse für alle Interessierten zum Nulltarif anzubieten. Dabei richtet man sich vor allem an Privatpersonen, implizit auch an

\footnotetext{
${ }^{2}$ Die Zahl wurde genannt bei einem Vortrag von Michaela Laemmler von SAP am 4. Dezember 2014 auf der Online Educa in Berlin.
} 


\section{Take the world's best courses,} online, for free.

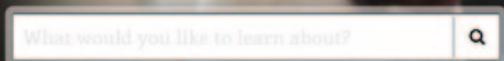

Join $9,968,267$ Courserians Learn from 8,4 courses, from our 114 partners. How it works $)$
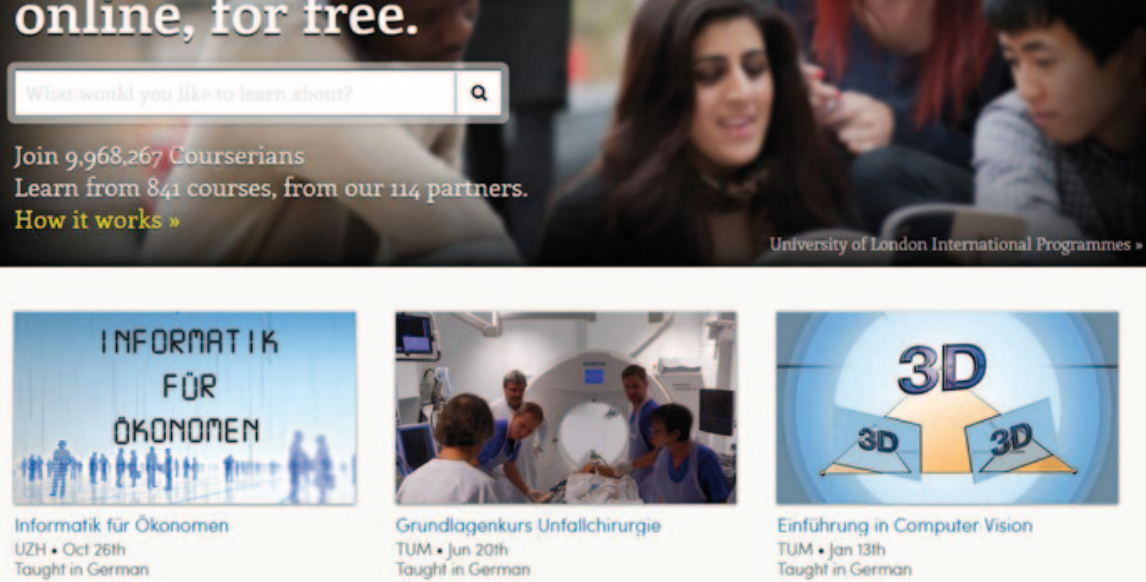

Abb. Snapshot von der Coursera-Website

Mitarbeitende: „We aim to empower people with education that will improve their lives, the lives of their families, and the communities they live in." (Website Coursera) Güntert (2013) ergänzt in zweideutiger Weise: „In Bezug auf Geschäftsmodelle arbeiten Organisationen wie Coursera daran, dass zum Abschluss eines MOOC Credits gegen Bezahlung erworben werden können."

Tim Kaltenborn zufolge können Firmen Marketing treiben, indem „sie eigene Filme als Case Studies zur Verfügung stellen - und sich so innerhalb eines OnlineLehrgangs selber als attraktive Marken oder Arbeitgeber zeigen können“ (Güntert 2013). Es ergeben sich zudem Chancen für Unis, die sich in globalen Rankings messen und versuchen, an die weltweit besten Talente zu kommen: „Im zunehmenden Wettbewerb in der Hochschullandschaft können MOOCs helfen, ausländische Studenten zu akquirieren. Inspirierende Professoren, die etwa in China oder im Mittleren Osten bei Studenten sehr gefragt sind, können eine Schaufensterwirkung entfalten.“ (Güntert 2013)

\section{MOOCs für die Wirtschaftsinformatik}

Bei einer Sichtung des MOOC-Angebots zur WI im englischen und deutschen Sprachraum muss man die unterschiedlichen Begriffe für die Disziplin berücksichtigen. ${ }^{3}$ Zudem sind die Teilgebiete der WI einzubeziehen. Allerdings sind deren viele, und je nach Hochschule und Land differieren die Zuordnungen. Nicht zuletzt ist es

\footnotetext{
${ }^{3}$ Informationsmanagement ist je nach Sichtweise ein Teilbereich oder weitgehend deckungsgleich mit der Wirtschaftsinformatik. Im Englischen existiert entsprechend der Begriff ,information management“.
} 
sinnvoll, Kurse in BWL und Informatik für die Untersuchung zuzulassen. Dadurch erweitert sich der Suchraum erheblich. Im Sinne einer ersten Annäherung soll der Gegenstandsbereich der WI weit gefasst werden. Es werden aus Platzgründen lediglich die genannten deutschen Plattformen und Coursera unter die Lupe genommen und beispielhaft Kurse - mit Semesterangabe oder Startdatum - genannt.

\subsection{Angebote aus der und für die Wirtschaftsinformatik}

Die IMC AG ist aus der IDS Scheer Consulting GmbH entstanden, einem Beratungsunternehmen von August-Wilhelm Scheer, einem Wirtschaftsinformatiker. Dies schlägt sich im Angebot der OpenCourseWorld nieder. So finden sich mit Stand September 2014 die Kurse „How to Create a Windows 8 App“ (diverse Partner, u. a. Microsoft und DFKI), „Business Process Management (BPM)“ (diverse Partner, Universität des Saarlandes und DFKI) und „UX - Design for Business“ (Fraunhofer IESE). Angekündigt werden „eBusiness für KMU“ (diverse Partner, u. a. Institut für kybernetisches Planen und Bauen, malerstammdaten.de und Fritz Stenger), „IT Sicherheit“ [!] (TU Darmstadt) und „Mobile Computing“ (Universität des Saarlandes). Es stechen falsche Schreibweisen bei Titeln und Beschreibungen ins Auge.

Das HPI-Portal ist auf die Informatik ausgerichtet. „Anhand von Lernvideos, interaktiven Selbsttests und praktischen Aufgaben werden vielfältige Themen der IT-Technologie vermittelt.“ (Website openHPI) Im Herbst 2014 sind Eigenproduktionen wie „In-Memory Data Management (2014) - Implications on Enterprise Systems" und „Einführung in die Internetsicherheit" gelistet. Man kann ferner bereits abgeschlossene Kurse durcharbeiten, allerdings ohne ein Zertifikat zu erwerben. Beispiele für solche Kurse sind „Business Process Modeling and Analysis (2013)“" und „Web-Technologien 2013“. Das Derivat openSAP wird wegen der Spezialisierung auf SAP-Produkte nicht weiter thematisiert. Von einer hohen Relevanz für die WI ist auszugehen.

iversity bietet in den Wirtschaftswissenschaften „Internationales Agrarmanagement" und „Einführung in die Betriebswirtschaftslehre“ (beide 14. Oktober 2014) sowie in den MINT-Fächern „Algorithmen und Datenstrukturen“ (14. Oktober 2014) und „Web Engineering“ (diverse Kurse seit 2014) an. Insofern E-Learning auch aus der Wirtschaftsinformatik heraus betrieben wird, sind die Kurse „Gamification Design Online Course“ (Beginn: 23. Juli 2014) und „Serious Game Design and Development" (13. Januar 2015) relevant.

Die UZH verantwortet den MOOC „Informatik für Ökonomen“ (26. Oktober 2014). Abgewickelt wird der Kurs über Coursera. Ziel ist es, ,eine Basis zu vermitteln, um unsere informationstechnisch gesteuerte Welt zu verstehen“ (Website Coursera). Ebenfalls auf der Plattform sind Kurse wie „Einführung in Computer Vision“ (TU München, 13. Januar 2014), „Social Network Analysis“ (University of Michigan, 6. Oktober 2014) und - durchaus selbstreferenziell - „Giving 2.0: The MOOC““ (Stanford University, 6. Oktober 2014).

Zudem spricht man u. a. von „,business information technology“, „,commercial information technology“, „,information systems“, „,business information systems“ und „,business informatics“. 


\subsection{Anrechenbarkeit und Verwertbarkeit}

MOOCs bieten nach Wedekind $(2013,56)$,inhaltliche Einzelangebote, aber kein Curriculum bis zum Erwerb eines akademischen Grades“. Das wird sich vermutlich bis auf Weiteres nicht ändern. Zu stark dürften bürokratische Widerstände und individuelle Vorbehalte sein. Auch die seriöse Bewertbarkeit steht in Frage. Ein möglicher Ansatz ist, die Abschlussprüfungen in Präsenz durchzuführen. iversity wirbt explizit mit der Anrechenbarkeit von Kursen: „Bei den Kursen von einigen dieser Hochschulen können die Teilnehmer Leistungspunkte nach dem ECTS-System erwerben, die für das Studium angerechnet werden können, was sonst bisher auf keiner anderen MOOC-Plattform möglich ist.“ (Website iversity) Zu den ECTS-Kursen gehören „Einführung in die Betriebswirtschaftslehre“ (Prof. Frank Piller, 14. Oktober 2014) und „Algorithmen und Datenstrukturen“ (Prof. Dr. Oliver Vornberger, Nils Haldenwang und Dr. Nicolas Neubauer, 14. Oktober 2014). Man kann also ECTS-Punkte für Informatik und WI sammeln. Substituiert die Hochschule klassische Kurse durch MOOCs, liegt diese Lösung nahe. Der Verantwortliche mag geschlossene Gruppen einrichten, für die spezifische Teilnahmebedingungen gelten. Alternativ kann er keine ECTS-, sondern Bonuspunkte verteilen.

\subsection{Chancen und Risiken}

An manchen der genannten MOOCs sind nicht nur Hochschulen beteiligt, sondern auch Unternehmen. Dies bietet Chancen und Risiken. Es ist sicherlich sinnvoll, dass Microsoft in ein Angebot involviert ist, das sich auf ein Produkt des Hauses bezieht, und SAP wird kompetent in Bezug auf SAP-Produkte sein. Auch die Fallbeispiele, die Betriebe beisteuern, sind von Nutzen. Problematisch wird es, wenn diese die Fachinhalte kontrollieren, wenn bestimmte Aspekte bevorzugt und andere vernachlässigt werden.

Chancen und Risiken entstehen auch für (Wirtschafts-)Informatikdozierende. Diese können stark repetitive und belastende Prozesse zurückdrängen, indem sie sich der Grundlagenvorlesungen in überfüllten Hörsälen entledigen, und interessante ergänzende Inhalte hinzunehmen. Sie vermögen mehrheitlich ihre Kurse selbst zu produzieren. Fragen müssen sie sich, ob ihr Auftritt, ihre Sprache und ihre Sprechweise den medialen Anforderungen genügen und sie sich durch die virtuellen Konserven überflüssig machen. Anreize und Regelungen der Hochschulen sind diesbezüglich dringend erforderlich.

$\mathrm{Zu}$ den Risiken gehört, dass nicht klar ist, ob MOOCs sich etablieren und Mittler sich halten. Zudem können sich die Geschäftsmodelle und mit ihnen die Kosten ändern. Wer als Lehrverantwortlicher auf Kurse und Plattformen setzt, kann sich demnach allenfalls kurz- oder mittelfristig auf die Existenz und Konsistenz verlassen. Bildungsmaßnahmen und Curricula müssen aber mittel- bis langfristig geplant werden. Oft werden MOOCs auch recht spontan angekündigt. Hier wäre es zielführend, mit dem Ersteller zusammenzuarbeiten.

Für Chancen und Risiken interessieren sich u. a. Technik- oder Technologiefolgenabschätzung (Technology Assessment, kurz TA) und Informationsethik. TA-SWISS (www.ta-swiss.ch) hat 2014 im WWW eine Vorstudie zu MOOCs ausge- 
schrieben (vgl. Website TA-SWISS). In Erfahrung gebracht werden soll, ob sich die Politik der neuen Lehr- und Lernformen annehmen muss und welche Konsequenzen für Gesellschaft und Bildung man vergegenwärtigen muss. Die Informationsethik hat die Moral der Informationsgesellschaft zum Gegenstand (Bendel 2013) und untersucht u. a. die Informationsgerechtigkeit. MOOCs können diese befördern, da sie sich prinzipiell an alle Interessierten richten, können aber genauso neue Gräben aufreißen, da sich ihnen - wie gezeigt - eher gut Ausgebildete zuwenden und auch in den 2010er-Jahren nicht alle Zugang zum Internet (und damit Informationsfreiheit in diesem Sinne) haben.

\section{Ein neuerliches Versprechen}

MOOCs sind ein neuerliches Versprechen der E-Learning-Protagonisten, das vermutlich teilweise eingelöst werden kann und teilweise gebrochen werden muss. Allein der Umstand, dass Hunderttausende auf diese Weise weitergebildet wurden, ist ein nicht wegzudiskutierender Erfolg, und Experten halten MOOCs für einen der wichtigsten Trends für die Zukunft (vgl. Goertz 2014, 14). Motivationseinbrüche und Abbrecherquoten sind indes ebenfalls Realität (vgl. Liyanagunawardena et al. 2014, 95 ff.). Nach ein paar Jahren ist vielleicht Erde verbrannt und Ernüchterung eingekehrt. Manche werden sich mit Vergnügen, andere mit Schrecken erinnern. Und einige werden sich weiterqualifiziert, Chancen ergriffen, die sonst nicht vorhanden gewesen wären, und eine Karriere darauf aufgebaut haben.

Es haben sich genügend virtuelle Optionen im WI-Bereich herausgebildet. Dies kommt nicht zuletzt dadurch, dass manche (gerade deutsche) Anbieter eine Nähe zu Informatik und Wirtschaftsinformatik haben (vgl. Website OpenCourseWorld). Sicherlich spielt auch eine Rolle, dass IT- und Businessthemen generell gut für E-Learning-Anwendungen geeignet sind und von jeher gerne angeboten und gut nachgefragt werden. ${ }^{4}$ Eine Umwälzung des Hochschulbetriebs durch MOOCs ist nicht zu erkennen, ebenso wenig eine Veränderung des Fachs.

Für die Studierenden ist es schwierig, zusätzlichen Stoff in ihrer grundständigen Ausbildung unterzubringen. Die konsequente Verknüpfung mit anrechenbaren Punkten könnte helfen, auch bei der organisierten Weiterbildung. Für die selbstverantwortete scheint es keine Hindernisse zu geben. Für die Dozierenden existieren tendenziell ungenügende Anreize, um großen individuellen Aufwand bei der Kurserstellung zu treiben. Nur wenn ihr Arbeitgeber hinter ihnen steht, wird sich etwas bewegen. Womöglich sind SPOCs, die weniger Mühe und Kosten bedeuten, eine Lösung. Manche Dozierende werden Angst haben, sich selbst abzuschaffen. Wirtschaftsinformatiker dürften für diese Thematik ein besonderes Gespür haben.

\footnotetext{
${ }^{4}$ Ein Grund ist, dass sich Softwarenutzung gut in Simulationen bzw. Animationen und mit Screenrecording abbilden lässt, und dass man Berechnungen gut in E-Learning-Umgebungen integrieren kann.
} 


\section{Literatur}

Becker M, Rojas R (2014) MOOCs statt Hörsaal: Der Unterricht im Zeitalter seiner technischen Reproduzierbarkeit. Heise Zeitschriften Verlag, Hannover

Bendel O (2011) Lernen von den Kleinen: Neues und Altes zu Mobile Learning. UnternehmerZeitung 4(17):32-33

Bendel O (2013) Die Medizinethik in der Informationsgesellschaft: Überlegungen zur Stellung der Informationsethik. Informatik-Spektrum 6(36):530-535

Bendel O (2014) Bildung für die Massen - Chancen und Risiken von MOOCs. Netzwoche 20:22

Bendel O, Stoller-Schai D (2001) E-Learning. In: Mertens P, Back A, Becker J et al (Hrsg) Lexikon der Wirtschaftsinformatik. 4., vollst. neu bearbeit. u. erweit. Aufl. Springer, Berlin, S. 164-165

Colman D (2013) MOOC Interrupted: Top 10 Reasons Our Readers Didn’t Finish a Massive Open Online Course. Open Culture, 5. April 2013. http://www.openculture.com/2013/04/10_reasons_you_didnt_ complete a mooc.html. Zugegriffen: 23. Okt. 2014

Goertz L (2014) Digitales Lernen adaptiv: Technische und didaktische Potenziale für die Weiterbildung der Zukunft. Bertelsmann Stiftung, Gütersloh. http://www.bertelsmann-stiftung.de/fileadmin/files/ BSt/Publikationen/GrauePublikationen/LL_GP_DigitalesLernen_final_2014.pdf. Zugegriffen: 6. Dez. 2014

Güntert A (2013) Weiterbildung: YouTube cum laude. bilanz.ch, 13. September 2013. http://www.bilanz. ch/ausbildung/weiterbildung-youtube-cum-laude. Zugegriffen: 23. Okt. 2014

Kandzia P-T (1999) VIROR - Die virtuelle Hochschule Oberrhein. In: Beiersdörfer K, Engels G, Schäfer W (Hrsg) Informatik'99 (Informatik aktuell 1999). Springer, Heidelberg, S. 60-69

Liyanagunawardena TR, Parslow P, Williams SA (2014) Dropout: MOOC Participants' Perspective. Cress U, Kloos CD (Hrsg.) (2014) EMOOCs 2014. Proceedings of the European MOOC Stakeholder Summit 2014. eLearning Papers, Brüssel, S. 95-100. http://www.emoocs2014.eu/sites/default/files/Proceedings-Moocs-Summit-2014.pdf. Zugegriffen: 23. Okt. 2014

Schmidt M (2013) Digitale Vorlesungshäppchen revolutionieren die Bildung. ZEIT ONLINE, 6. Juni 2013. http:/www.zeit.de/studium/uni-leben/2013-06/mooc-deutschland-iversity-vorlesungen-internet. Zugegriffen: 23. Okt. 2014

Schulmeister R (2013) Der Beginn und das Ende von OPEN: Chronologie der MOOC-Entwicklung. In: Schulmeister R (Hrsg) MOOCs - Massive Open Online Courses: Offene Bildung oder Geschäftsmodell? Waxmann, Münster, S. 17-59

Wedekind J (2013) MOOCs - eine Herausforderung für die Hochschulen. In: Reinmann G, Ebner M, Schön S (Hrsg) Hochschuldidaktik im Zeichen von Heterogenität und Vielfalt. BIMS e. V., Bad Reichenhall, S. 45-62

Website Coursera. http://www.coursera.org. Zugegriffen: 23. Okt. 2014

Website iversity. http://www.iversity.org. Zugegriffen: 23. Okt. 2014

Website OpenCourseWorld. http://www.opencourseworld.de. Zugegriffen: 23. Okt 2014

Website openHPI. https://open.hpi.de. Zugegriffen: 23. Okt. 2014

Website openSAP. https://open.sap.com. Zugegriffen: 6. Dez. 2014

Website TA-SWISS. http://www.ta-swiss.ch. Zugegriffen: 23. Okt. 2014 